\title{
Surface Self-Diffusion and Mean Displacement of Hydrogen on Graphite and a PEM Fuel Cell Catalyst Support
}

\author{
Ole-Erich Haas, ${ }^{\star \dagger}$ Jean Marc Simon, ${ }^{\ddagger}$ and Signe Kjelstrup ${ }^{\ddagger}, \$$ \\ Department of Chemistry, Norwegian University of Science and Technology, NO 7491 Trondheim, Norway, Institut \\ Carnot de Bourgogne, UMR-5209 CNRS-Université de Bourgogne, 9 av. A. Savary, 21000 Dijon, France, and \\ Department of Process and Energy, Technical University of Delft, 2628 EV Delft, The Netherlands
}

Received: March 19, 2009; Revised Manuscript Received: September 9, 2009

\begin{abstract}
Quasielastic neutron scattering (QENS) measurements together with equilibrium molecular dynamic (EMD) simulations have been performed to investigate the surface interaction between hydrogen molecules and a carbon material commonly used in polymer electrolyte membrane fuel cells (PEMFC), called XC-72. Half a monolayer of molecular hydrogen was adsorbed on to the carbon material at $2 \mathrm{~K}$. QENS spectra were recorded at the time-of-flight spectrometer IN5 at 40, 45, 50, 60, 70, 80, and $90 \mathrm{~K}$. Simultaneously the pressure was measured as a function of time to monitor the equilibrium surface coverage at each temperature. By using the Chudley and Elliott model for jump diffusion we found the diffusion coefficient at each temperature. At 350 $\mathrm{K}$, a typical fuel cell temperature, the temperature function was extrapolated to a self-diffusion coefficient of $2.3 \times 10^{-7} \mathrm{~m}^{2} / \mathrm{s}$. We simulated graphite in contact with hydrogen molecules using EMD simulation. We simulated the system at different temperatures from 70 to $350 \mathrm{~K}$ in 20 deg intervals and for five numbers of $\mathrm{H}_{2}$ molecules $N_{\mathrm{H}_{2}}=50,100,150,200$, and 300 . The graphite was made of 9 sheets of graphene in a sandwich. The surface self-diffusion was found from the mean-square displacement, and the values from EMD simulations are the same order of magnitude as the experimental values at $90 \mathrm{~K}$, but systematically higher, probably due to the ideal surface. From EMD simulation, we also calculated the average time between adsorption and desorption events on the surface. This was used to find the mean displacement of the hydrogen molecules between adsorption and desorption. This result showed that $\mathrm{H}_{2}$ molecules can move $80 \AA$ at ambient temperatures and pressures, along the surface. Using these data, we conclude that catalyst support material in PEMFC contributes to the transport of reactant.
\end{abstract}

\section{Introduction}

One of the limiting factors for a realization of the polymer electrolyte membrane fuel cell as a energy converter may be the catalyst utilization in the cell. The impressive catalyst loading that now has been reached in many recent works, ${ }^{1,2} 0.02-0.09$ $\mathrm{mg} / \mathrm{cm}^{2}$, may still not be sufficient for an introduction of fuel cells in the transport sector. The amount of catalyst in the world is limited, so the material available must be efficiently used. Work that can help reduce the loading, and reduce mass transfer limitations in the cell is therefore important. The present work can be seen in this context.

The cell consists of two catalytic layers, a few micrometers thick, separated by a proton conducting water-filled polymer electrolyte membrane. A detailed description is given by Larminie et al. and Costamagna et al. ${ }^{3,4}$ The reactive sites in the catalytic layers are located on Pt or Pt alloy particles, with diameters of typically $2-10 \mathrm{~nm} .^{5}$ The particles must be in contact with the membrane as well as an electronic conductor, for instance carbon black (XC-72).$^{6-9}$ The catalytic layers are further supported by a layer made of carbon paper or cloth. This layer, as well as the catalytic layer, are both porous. They allow reactant gases $\mathrm{H}_{2}$ and $\mathrm{O}_{2}$ to diffuse to the catalytic sites. The question to be examined in this article is the mechanism

\footnotetext{
* To whom correspondence should be addressed.

$\uparrow$ Norwegian University of Science and Technology.

* Institut Carnot de Bourgogne.

$\S$ Technical University of Delft.
}

of hydrogen's transport. Which path does hydrogen take on its way to the catalytic site, and what is the corresponding transport parameter?

There is contact between the gas, the catalyst, and the ionic conductor at the three phase contact line between the phases. These lines provide a rather limited number of sites. Direct access of hydrogen gas to the three-phase contact line via the gas phase is also impossible: Gas molecules cannot go directly to a contact line from the gas phase as this would lead to an infinite velocity of the gas close to the contact line. ${ }^{10}$ A supply of gas to the active sites must then come via the carbon surface or membrane surface in contact with Pt. Accordingly, Meland et al. ${ }^{11}$ proposed that the path to the catalytic site is via the relevant surfaces. Some evidence to support this hypothesis was obtained by impedance spectroscopy. ${ }^{11-13}$ A surface diffusion coefficient for hydrogen on carbon was estimated to be $1 \times$ $10^{-7} \mathrm{~m}^{2} / \mathrm{s} .{ }^{12}$

These investigations indicate that surface diffusion can be essential for supply of reactants to the catalytic sites. It is then important to have precise knowledge of its value and variation. It is the aim of the present work to obtain such knowledge. Some initial studies of hydrogen diffusion on carbon have already been done using quasielastic neutron scattering experiments, by us ${ }^{14}$ and others. ${ }^{15,16}$ Adsorption of $\mathrm{H}_{2}$ on carbon materials has been studied by many authors. ${ }^{17-25}$ But these investigations have been directed toward use of carbon materials as hydrogen storage materials, and not as catalyst support in PEMFCs. 
We give here further evidence supporting the idea that significant hydrogen transport can take place along carbon or graphitic surfaces. Quasielastic neutron scattering (QENS) experiments are well suited to find surface diffusion of molecular hydrogen on carbon materials $1^{5,16,26-29}$ and we shall use this technique again to study carbon black, XC-72, reporting new data and improvements on the method. ${ }^{14}$ The new experiments give a more accurate determination than reported before, ${ }^{14}$ another surface concentration at $2 \mathrm{~K}$, and values for additional temperatures. The variation in the surface self-diffusion coefficient $D_{\|}^{\mathrm{s}}$ with surface concentration and temperature shall be presented for temperatures between 40 and $90 \mathrm{~K}$.

The experimental technique is supplemented with equilibrium molecular dynamics simulations of hydrogen on graphite between 70 and $350 \mathrm{~K}$, in order to reveal underlying molecular mechanisms. We shall see that there can be a significant hydrogen transport to the Pt catalyst particles via the graphite support. This supports the proposition that hydrogen transport to the anode in a PEMFC may take place via the carbon surface.

To distinguish surface diffusion from diffusion in the homogeneous phase from each other, we shall use the symbol $D_{\|}^{\mathrm{s}}$ for diffusion parallel to the surface and $D_{\perp}^{\mathrm{s}}$ for diffusion into the surface, while $D$ shall be used for the gas phase only.

\section{Experimental Section}

Sample Characterization and Preparation. The sample used in this experiment is a commercial carbon black, XC-72, from Cabot Corp, that was described before. ${ }^{14}$ From BET measurements the surface area of XC-72 $\left(A_{\mathrm{XC}-72}\right)$ was found to be $204.3 \mathrm{~m}^{2} / \mathrm{g}$. The sample $\left(m_{\mathrm{XC}-72}=8.1 \mathrm{~g}\right)$ was placed in a thin walled aluminum cylinder and evacuated for $6 \mathrm{~h}$ in order to remove water. The number of moles needed to form half a monolayer of hydrogen $\left(n_{\mathrm{H}_{2}}^{\text {total }}=0.0086 \mathrm{mmol}\right)$ was estimated from the BET surface area of the sample $\left(A_{\mathrm{XC}-72}\right)$ and the molecular area of hydrogen found in literature $\left(A_{\mathrm{H}_{2}}\right)$ equal to $15.6 \AA^{2}{ }^{15}$ To measure the number of moles needed to form half a monolayer, a second cylinder with a known volume of $1856 \mathrm{~cm}^{3}$ was pressurized until the pressure reached 85.63 Torr at room temperature. The pressure of $n$-hydrogen $\left(0.75 o-\mathrm{H}_{2}\right.$, $0.25 p-\mathrm{H}_{2}$ ) was measured with a Baratron pressure gauge at room temperature and hydrogen was introduced to the graphite in the aluminum cylinder at $2 \mathrm{~K}$ and the cell was then sealed. The hydrogen was left at $2 \mathrm{~K}$ to equilibrate with $p$-hydrogen before the measurements were started. The surface coverage and surface concentration were calculated from the pressure in the closed system measured at room temperature. From the ideal gas law, the volume of the system $(V)$, the pressure $(P)$, and the gas temperature $(T)$, we calculated the amount of hydrogen in the gas phase $\left(n_{\mathrm{H}_{2}}^{\mathrm{g}}\right)$. From knowledge of the total amount of $\mathrm{H}_{2}$ in the system $\left(n_{\mathrm{H}_{2}}^{\text {total }}\right)$, the BET area of the sample $\left(A_{\mathrm{XC}-72}\right)$, the mass of the sample $\left(m_{\mathrm{XC}-72}\right)$, Avogadro's number $\left(N_{\mathrm{A}}\right)$, and the area of an adsorbed hydrogen molecule $\left(A_{\mathrm{H}_{2}}\right)$, we found the number of surface molecules $\left(n_{\mathrm{H}_{2}}^{\mathrm{s}}\right)$ in moles, surface coverage $(\theta)$, and surface concentration $\left(c_{\mathrm{H}_{2}}^{\mathrm{s}}\right)$ as

$$
\begin{gathered}
n_{\mathrm{H}_{2}}^{\mathrm{s}}=n_{\mathrm{H}_{2}}^{\mathrm{total}}-n_{\mathrm{H}_{2}}^{\mathrm{g}}=n_{\mathrm{H}_{2}}^{\text {total }}-\frac{P V}{R T} \\
\theta=\frac{A_{\mathrm{H}_{2}} N_{\mathrm{A}} n_{\mathrm{H}_{2}}^{\mathrm{s}}}{A_{\mathrm{XC}-72} m_{\mathrm{XC}-72}} \\
c_{\mathrm{H}_{2}}^{\mathrm{s}}=\frac{n_{\mathrm{H}_{2}}^{\mathrm{s}}}{A_{\mathrm{XC}-72} m_{\mathrm{XC}-72}}
\end{gathered}
$$

where $R$ is the gas constant. This gives an improvement in the experimental setup compared to our earlier work. ${ }^{14}$ We can now correlate the surface concentration and the surface self-diffusion.

Quasielastic Neutron Scattering Experiments. The quasielastic neutron scattering (QENS) experiments were performed at the direct geometry time-of-flight spectrometer IN5, ILL, Grenoble, France, mainly as described before. ${ }^{14}$ The main improvement was that corrections were made for temperaturedependent background corrections. To estimate the resolution function a measurement of a cylindrical vanadium sample at $40 \mathrm{~K}$ was preformed. ${ }^{30}$ The background was measured with the sample container filled with XC-72 at 40, 45, 50, 60, 70, 80, and $90 \mathrm{~K}$. Measurements to estimate the diffusion were conducted at 40, 45, 50, 60, 70, 80, and $90 \mathrm{~K}$. To verify that the system was in equilibrium and stable during the measurements, the pressure was constantly measured, giving constant information on the surface concentration and surface coverage from eq 1.

Data collected from the QENS measurements were first normalized versus the monitor. This was done to remove changes in intensities between spectra due to variations in the incident neutron beam. To correct for variations in detector efficiencies a measurement of a cylindrical vanadium sample at $40 \mathrm{~K}$ was conducted. Then the spectra were normalized to the vanadium measurements to correct the intensity at each momentum transfer $Q$ value, $\mathbf{Q}$ being the scattering vector. Further, the measured spectra were divided into regions in $Q$, $\omega$ (energy transfer) space to get constant $Q$ values by rebinning over $d Q=0.05 \AA^{-1}$ to enhance the statistics. This was done with LAMP, a program from ILL based on the IDL language. The background measurements of pure XC-72 were treated the same way and subtracted from the measurements of XC-72 with hydrogen. After this treatment the $S(\omega)$ plots were fitted with a Lorentzian function to estimate quasielastic broadening as a function of $Q, f(Q)$. The fit of the Lorentzian was done in QENS_fit, a fitting procedure within the LAMP program. In the QENS_fit program a measurement of the vanadium sample at $40 \mathrm{~K}$ was used as the resolution function. The fit to the broadening as a function of $Q$ was done with the Chudley and Elliott model that describes the movement of a single molecule as it jumps between different sites in a lattice or between cages made of other molecules. ${ }^{30}$ The smallest jump length $l_{0}$ was assumed to be identical for all sites whereas the jump directions are random. It is assumed that the molecules remain at the surface during a time $\tau_{0}$, the residence time, before moving again. Using the Einstein-Smoluchowski equation, see i.e. ref 31 , the surface diffusion coefficient is then

$$
D_{\|}^{\mathrm{s}}=\frac{l_{0}^{2}}{\tau_{0}}
$$

The subscript indicates that diffusion takes place along the surface, and the superscript s that the value is specific to the surface.

Using a model of the broadening function $f(Q)$ described before, ${ }^{14}$ we were able to find $D_{\|}^{\text {s }}$ by fitting data to the following form:

$$
\frac{\hbar}{f(Q)}=\tau_{0}+\frac{\tau_{0}}{l_{0}^{2} Q^{2}}=\tau_{0}+\frac{1}{D_{\|}^{\mathrm{s}} Q^{2}}
$$

where $\hbar$ is the reduced Planck constant. 
TABLE 1: Parameters Found from the QENS Experiment

Data form QENS with 0.5 monolayer of $\mathrm{H}_{2}$ at $2 \mathrm{~K}$

\begin{tabular}{ccccc}
$c_{\mathrm{H}_{2}}^{\mathrm{s}}\left(\mathrm{mmol} / \mathrm{m}^{2}\right)$ & temp $(\mathrm{K})$ & $10^{-12} \tau(\mathrm{s})$ & $10^{-10} l_{0}(\mathrm{~m})$ & $10^{-8} D_{\|}^{\mathrm{s}}\left(\mathrm{m}^{2} / \mathrm{s}\right)$ \\
\hline 0.0051 & 40 & $1.21 \pm 0.07$ & $1.20 \pm 0.05$ & $1.18 \pm 0.07$ \\
0.0050 & 45 & $1.22 \pm 0.06$ & $1.37 \pm 0.05$ & $1.53 \pm 0.09$ \\
0.0046 & 50 & $1.23 \pm 0.05$ & $1.50 \pm 0.06$ & $1.8 \pm 0.1$ \\
0.0029 & 60 & $1.26 \pm 0.05$ & $2.15 \pm 0.01$ & $3.7 \pm 0.5$ \\
0.0023 & 70 & $1.35 \pm 0.04$ & $2.52 \pm 0.01$ & $4.7 \pm 0.5$ \\
0.0016 & 80 & $1.30 \pm 0.06$ & $2.79 \pm 0.02$ & $6.0 \pm 0.9$ \\
0.0012 & 90 & $1.1 \pm 0.1$ & $2.92 \pm 0.05$ & $8 \pm 3$ \\
\hline
\end{tabular}

Data form QENS with 1 monolayer of $\mathrm{H}_{2}$ at $2 \mathrm{~K}^{14}$

\begin{tabular}{cccc}
\hline temp $(\mathrm{K})$ & $10^{-12} \tau(\mathrm{s})$ & $10^{-10} l_{0}(\mathrm{~m})$ & $10^{-8} D_{\|}^{\mathrm{s}}\left(\mathrm{m}^{2} / \mathrm{s}\right)$ \\
\hline 40 & $1.5 \pm 0.2$ & $1.3 \pm 0.1$ & $1.1 \pm 0.1$ \\
50 & $1.2 \pm 0.1$ & $1.6 \pm 0.1$ & $2.1 \pm 0.3$ \\
60 & $1.0 \pm 0.1$ & $1.7 \pm 0.2$ & $3.0 \pm 0.5$ \\
70 & $0.9 \pm 0.1$ & $1.8 \pm 0.2$ & $3.7 \pm 0.7$
\end{tabular}

By plotting the logarithm of the diffusion coefficients found for each temperature versus $T^{-1}\left(\mathrm{~K}^{-1}\right)$, we obtained an Arrhenius plot. This was used to estimate the temperature dependence of the diffusion coefficient according to

$$
D_{\text {॥ }}^{\mathrm{s}}(T)=D_{0} \exp \left(\frac{-E}{k_{\mathrm{B}} T}\right)
$$

Here $D_{0}$ is the pre-exponential factor, $k_{\mathrm{B}}$ is the Boltzmann constant, and $E$ is the activation energy. We will compare the results from QENS with results from EMD.

\section{Equilibrium Molecular Dynamics Simulations}

The System. The EMD system was built of an infinite sheet of crystalline graphite in contact with hydrogen molecules, $\mathrm{H}_{2}$. The graphite has a hexagonal crystallographic structure with space group $P 6_{3} / m m c$ without any defects. The crystal is made from 9 sheets of graphene and contains 5184 carbon atoms. Following the crystallographic structure, the graphene sheets are oriented in our simulation box such that the surfaces of the sheets are perpendicular to the $\mathbf{z}$ direction. The size of the crystal is $44.208 \AA$ and $34.0313 \AA$ in $\mathbf{x}$ and $\mathbf{y}$ directions, respectively, and about $30 \AA$ in the $\mathbf{z}$ direction. ${ }^{32}$ Periodic boundary conditions are applied in $\mathbf{x}$ and $\mathbf{y}$ directions in the limits of the crystal and in the $\mathbf{z}$ direction at $95.254 \AA$ from the center of the crystal. This symmetry gives in effect an infinite succession of two infinite graphite crystal surfaces separated by a gaseous zone of about $160 \AA$ in the $\mathbf{z}$ direction, where the $\mathrm{H}_{2}$ molecules are located. The model uses an atomic description of the graphite and hydrogen. The atoms interact through intermolecular and intramolecular potentials. The intramolecular potentials are given in ref 33, Table 1, and eqs $2-4$. The intermolecular potential is the Lennard-Jones potential. ${ }^{34}$ The potential parameters for the Lennard-Jones are taken from the MM2 force field, ${ }^{35}$ while the parameters of the intramolecular potentials come from the DREIDING force field ${ }^{36}$ for graphite and Delft Molecular Mechanics (DMM) force field ${ }^{33}$ for the hydrogen molecules. The parameters of these potentials were fitted as to give good agreement with experimental physical properties of a large variety of compounds mainly those including carbon and hydrogen, like hydrocarbons. They are well-known and widely used in molecular simulations.

Simulation Procedures. Five different systems were simulated to investigate the surface diffusion coefficient as a function of surface concentration. The surface concentration variation was implemented by simulating with five different global densities. The five different numbers of hydrogen molecules were $N_{\mathrm{H}_{2}}=50,100,150,200,300$. For each $N_{\mathrm{H}_{2}}$, simulations were preformed at 17 different temperatures, ranging from 70 to $350 \mathrm{~K}$ at $20 \mathrm{deg}$ intervals. Additional simulations with $\mathrm{N}_{\mathrm{H}_{2}}$ $=10$ were performed at $70,90,110,150,170,190$, and $230 \mathrm{~K}$ to investigate the surface dynamics at low density and low temperature. This gave a total of 82 simulations describing the dynamics on the graphite surface as a function of surface concentration and temperature. The volume of the total system subtracted by the volume of the graphite crystal gives the volume available to the $\mathrm{H}_{2}$ molecules in the gas phase.

The dynamics were obtained by integrating Newton's equations of motion, using the velocity Verlet algorithm. ${ }^{37}$ All simulations had time steps of $0.001 \mathrm{ps}$. The initial configuration was constructed by randomly distributing the $\mathrm{H}_{2}$ molecules in the gas phase. Molecules are located such that they do not overlap with each other or the graphite crystal. The system was stabilized during $400 \mathrm{ps}$ by fixing the temperature at an imposed value, using a simple rescaling of the velocities. When the system was in thermal equilibrium, the conditions of the simulations were changed to those of a microcanonical ensemble (constant energy). Trajectories of 1700 ps were then computed and analyzed. Variables were calculated every 10 time steps, averaged over $1 \mathrm{ps}$, and stored.

Surface Self-Diffusion Coefficient and Mean Displacement. Here we present how we have analyzed the EMD data to find the transport properties. From the EMD simulations all the movements of the particles are calculated and stored. These data give us access to the diffusivities of the $\mathrm{H}_{2}$ molecules. This diffusivity is known as the self-diffusion or the tracer diffusion. It can be derived from the perspective of random walk. ${ }^{34,38}$ In this paper we have investigated the transport along the graphite surface. On a flat graphite surface it can be assumed that the surface is homogeneous and that the steps in the random walk are uncorrelated. For random walk in two dimensions the meansquare displacement is then given by

$$
\left\langle r^{2}(t)\right\rangle=a_{x}^{2} n_{x}(t)+a_{y}^{2} n_{y}(t)
$$

where $\left\langle r^{2}(t)\right\rangle$ is the mean-square displacement, $a_{i}$ is the length moved by the particle in either the $x$ or $y$ direction, and $n_{x}(t)$ and $n_{y}(t)$ are the number of jumps done during the time $t$. If the system is homogeneous it can be assumed that $a=a_{x}=a_{y}$ and that $n(t)=n_{x}(t)+n_{y}(t)$ and eq 5 can be written as

$$
\left\langle r^{2}(t)\right\rangle=a^{2} n(t)
$$

The surface self-diffusion coefficient $D_{\|}^{\mathrm{s}}$ is given by

$$
\left\langle r^{2}(t)\right\rangle=\lim _{t \rightarrow \infty} 2 d D_{\|}^{\mathrm{s}} t
$$

which is possible to rearrange to get

$$
D_{\|}^{\mathrm{s}}=\lim _{t \rightarrow \infty}\left[\frac{\left\langle r^{2}(t)\right\rangle}{2 d t}\right]
$$

For surface self-diffusion the dimensionality $d$ is equal to 2 , the diffusion is independent of the nature of the jumps. For a 


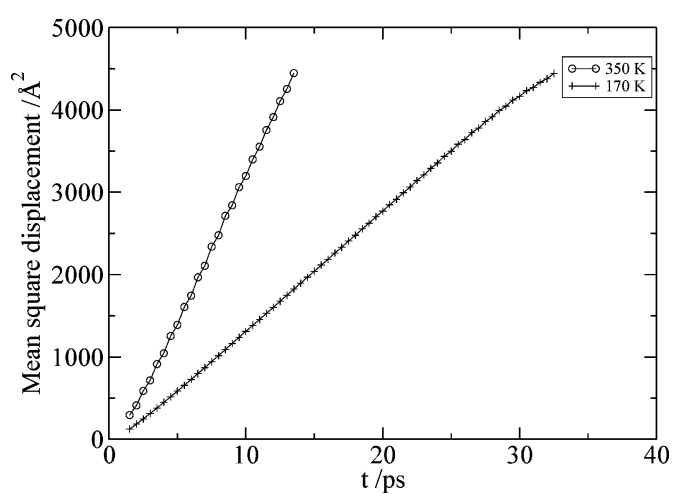

Figure 1. Examples of slopes of the mean-squared displacement of hydrogen molecules as a function of the time for 170 and $350 \mathrm{~K}$ with $N_{\mathrm{H}_{2}}=300$.

group of $N$ distinguishable particles eq 9 gives the surface selfdiffusion coefficient in the form

$$
D_{\|}^{\mathrm{s}}=\lim _{t \rightarrow \infty}\left[\frac{1}{2 d N t} \sum_{i=1}^{N}\left\langle\left|\vec{r}_{i}(t)-\vec{r}_{i}(0)\right|^{2}\right\rangle\right]
$$

where $\vec{r}_{i}(0)$ and $\vec{r}_{i}(t)$ are the positions of molecule $i$ at an initial time and after a time delay of $t$. This equation was used to extract the surface self-diffusion coefficient from the movements of each individual particle. Plots of $\left\langle r^{2}(t)\right\rangle$ as a function of $t$ are shown in Figure 1 below. The slope of these plots gives the $D_{\|}^{\text {s from }}$ the molecular dynamics simulation data. At equilibrium the time between an adsorption and desorption event for each molecule was calculated. These were next used to obtain the average time, $\langle t\rangle$, that the molecules spent on the graphite surface at each simulated temperature and surface concentration. From the values of $D_{\|}^{\mathrm{s}}$ and the average time on the surface, the mean displacement, $r_{\text {mean }}$, is given by

$$
r_{\text {mean }}=\sqrt{4 D_{\|}^{\mathrm{s}}\langle t\rangle}
$$

Here $r_{\text {mean }}$ is the mean displacement that the molecules have moved from the point they adsorb to the point they desorb. This value shall be used later to estimate the contribution of the graphite catalyst support on the transport of reactant to a catalyst particle.

\section{Results and Discussion}

Quasielastic Neutron Scattering Experiments. Figure 2a shows the fit of eq 3 to the experimental data at 40 and $90 \mathrm{~K}$, for $Q$ values from 0.45 to $1.35 \AA^{-1}$. The values of the surface self-diffusion coefficients, $D_{\|}^{\mathrm{s}}$, the residence times, $\tau_{0}$, and the jump lengths, $l_{0}$, from eq 3 are presented in Table 1 with the corresponding uncertainties. The $D_{\|}^{\mathrm{s}}$ values for $40,45,50,60$, 70,80 , and $90 \mathrm{~K}$ from eqs 3 are plotted in an Arrhenius plot shown in Figure 2b. From the slope of this plot, we determined the coefficients $D_{\| 0}^{\mathrm{s}}=(3.5 \pm 0.9) \times 10^{-7} \mathrm{~m}^{2} / \mathrm{s}$ and $E / k_{\mathrm{B}}=140$ $\pm 10 \mathrm{~K}(1160 \pm 80 \mathrm{~J} / \mathrm{mol})$ in eq 4 . For comparison, liquid hydrogen has the Arrhenius coefficients $D_{0}=(8.6 \pm 1.0) \times$ $10^{-8} \mathrm{~m}^{2} / \mathrm{s}$ and $E / k_{\mathrm{B}}=45 \pm 2 \mathrm{~K}(373 \pm 13 \mathrm{~J} / \mathrm{mol}) .{ }^{39}$ Using eq 4 , we estimated the self-diffusion coefficient at higher temperatures. At $350 \mathrm{~K}$, a typical fuel cell temperature, we found a self-diffusion coefficient of $2.3 \times 10^{-7} \mathrm{~m}^{2} / \mathrm{s}$.

Table 1 gives the results of the QENS experiments. Also the previous QENS results are presented for comparison. The two

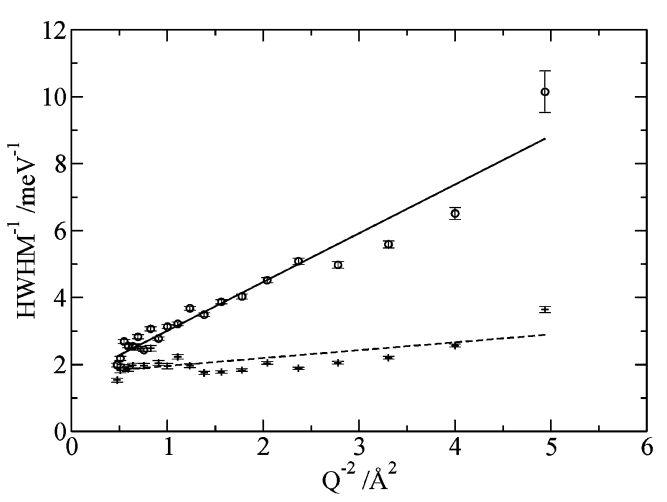

(a) Experimental data from QENS

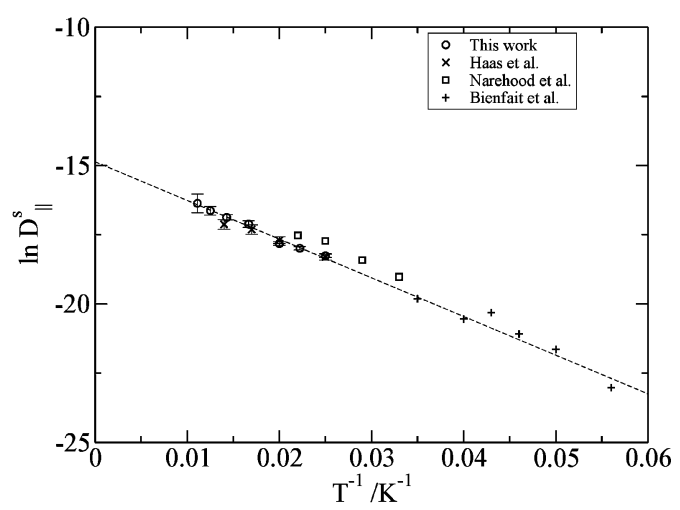

(b) Self diffusion coefficients from QENS

Figure 2. (a) Fit of eq 3 to the experimental data from the QENS measurements done at 40 and $90 \mathrm{~K}$. The dashed line is the fit to the data from $40 \mathrm{~K}$ and the full line is the fit to the data from $90 \mathrm{~K}$. (b) Natural logarithm of the self-diffusion coefficients found from our QENS experiments (this work and previous work). Data are plotted together with results from Narehood et al. and Bienfait et al. as a function of $T^{-1}\left(\mathrm{~K}^{-1}\right)$. The dashed line is the linear regression giving the Arrhenius coefficients $D_{\| 0}^{\mathrm{s}}$ and $E / k_{\mathrm{B}}$ from our QENS data.

sets of results are also compared in Figure $2 b$. We see that the new results can be given with uncertainties better than $\pm 5 \%$ below $70 \mathrm{~K}$. At 80 and $90 \mathrm{~K}$, the accuracy becomes less. A clear increase can now be seen in the diffusion coefficient with temperature, and this can be ascribed to an increased jump length, since the residence time is constant within the accuracy given. The new experiments for the diffusion coefficients agree in a qualitative way with the previous results. The accuracy in these experiments was smaller, however. They pointed to a reduction in residence time with temperature, but this is probably not real, as these data did not have temperature-dependent background corrections.

The new experiments are done with half a monolayer of hydrogen at $2 \mathrm{~K}$, while the previous ones were done with a full monolayer at $2 \mathrm{~K}$. This may explain why the first measured diffusion coefficients on the average are somewhat larger (a factor of 1.1-1.4) than the new results; the surface concentration must be larger in the last case, but it was then not determined. In a recent article Cabria et al. ${ }^{40}$ studied adsorption of $\mathrm{H}_{2}$ on carbon nanotubes using density functional theory (DFT). They found four different sites on a graphene layer with different binding energies. In a powder sample like here, the number of sites can be larger.

The temperature dependence of the two sets of data as found from eq 4 is also slightly different. We find 


$$
\begin{aligned}
D_{0.5 \mathrm{ML}}^{\mathrm{s}} & =(3.5 \pm 0.9) \times 10^{-7} \exp \left(\frac{-1.2 \pm 0.1 \mathrm{~kJ} / \mathrm{mol}}{R T}\right) \mathrm{m}^{2} / \mathrm{s} \\
D_{1 \mathrm{ML}}^{\mathrm{s}} & =(1.9 \pm 0.2) \times 10^{-7} \exp \left(\frac{-1.0 \pm 0.1 \mathrm{~kJ} / \mathrm{mol}}{R T}\right) \mathrm{m}^{2} / \mathrm{s}
\end{aligned}
$$

By extrapolation to $350 \mathrm{~K}$, we find $D_{0.5 \mathrm{ML}}^{\mathrm{s}}=2.3 \times 10^{-7} \mathrm{~m}^{2} / \mathrm{s}$ and $D_{1 \mathrm{ML}}^{\mathrm{s}}=1.4 \times 10^{-7} \mathrm{~m}^{2} / \mathrm{s}$. The effects of temperature and concentration are separable with the molecular dynamics simulation technique (see below).

Surface Concentration and Enthalpy and Entropy of Adsorption. The surface concentration, $c_{\mathrm{H}_{2}}^{\mathrm{s}}$, was calculated for each temperature investigated by QENS from the pressure measurements, using eq 1 . The measured pressure and the corresponding surface coverage, $\theta$, from eq 1 were first used to find the Langmuir constant, $K_{\mathrm{L}}$, from

$$
K_{\mathrm{L}}=\frac{\theta}{(1-\theta)} \frac{p^{0}}{p}
$$

The logarithm of $K_{\mathrm{L}}$ is shown in the upper part of Figure 3 as a function of the inverse of the temperature. A linear trend is found. This is remarkable since we only have one experimental point for each temperature. This shows that the adsorption isotherm from pressure measurement fits well with a Langmuir isotherm. The same was observed earlier with molecular dynamics simulations of hydrogen on graphite. ${ }^{41,42}$ These results are also shown in the same figure for comparison. The two lines give the enthalpy (the slope) $\Delta H$ and entropy (the intersection) $\Delta S$ of adsorption at zero loading.

$$
\begin{aligned}
\Delta H_{\text {exp }} & =-3.3 \mathrm{~kJ} / \mathrm{mol}, \Delta S_{\text {exp }}=-34 \mathrm{~J} / \mathrm{kmol} \\
\Delta H_{\mathrm{MD}} & =-6.0 \mathrm{~kJ} / \mathrm{mol}, \Delta S_{\mathrm{MD}}=-72 \mathrm{~J} / \mathrm{kmol}
\end{aligned}
$$

The results are of the same order of magnitude. The simulation of the pure graphite-hydrogen system gives values that are twice the values of the experimental ones, but both sets of data are in agreement with previous studies, both experimental and theoritical. ${ }^{28,40,43-48}$

Equilibrium Molecular Dynamics Simulations. The surface self-diffusion of hydrogen molecules on graphite, $D_{\|}^{\mathrm{s}}$, was obtained from equilibrium molecular dynamics simulations. The value of $D_{\|}^{\mathrm{s}}$ was obtained by linear fit to the slope of the meansquared displacement from eq 9, as shown in Figure 1. $D_{\|}^{\text {s }}$ for temperatures from 70 to $350 \mathrm{~K}$ with $20 \mathrm{deg}$ intervals and at 82 different surface concentrations were found in this manner and plotted as function of $c_{\mathrm{H}_{2}}^{\mathrm{s}}$ in Figure $4 \mathrm{a}$. This plot shows that all data follow a similar trend regardless of temperature. In Figure $4 \mathrm{~b}$ we plotted the experimental results reported above. We observed that $D_{\|}^{\mathrm{S}}$ has the same $c_{\mathrm{H}_{2}}^{\mathrm{s}}$ dependence as the data from QENS.

Figure 5 gives $D_{\|}^{\mathrm{s}}$ at 70, 90, 110, 150, 170, 190, 230, and $350 \mathrm{~K}$ as a function of $c_{\mathrm{H}_{2}}^{\mathrm{s}}{ }^{-1}$. This plot is made to investigate the prediction of kinetic theory for surface diffusion. According to this theory, the diffusion coefficient varies with $c_{\mathrm{H}_{2}}^{\mathrm{S}}{ }^{-1}$ below approximately $1000 \mathrm{~m}^{2} / \mathrm{mmol}^{38,49}$ Our data start to deviate from a linear relation above $1000 \mathrm{~m}^{2} / \mathrm{mmol}$. At this concentration the molecules on the surface are not colliding with each other anymore, and the $D_{\|}^{\mathrm{s}}$ approaches a (temperature dependent) maximum value. At temperatures from $190 \mathrm{~K}$ and up, and with a global number of $N_{\mathrm{H}_{2}}=10$, the density on the graphite surface is very low, and the uncertainties both in $D_{\|}^{\mathrm{s}}$ values and in the

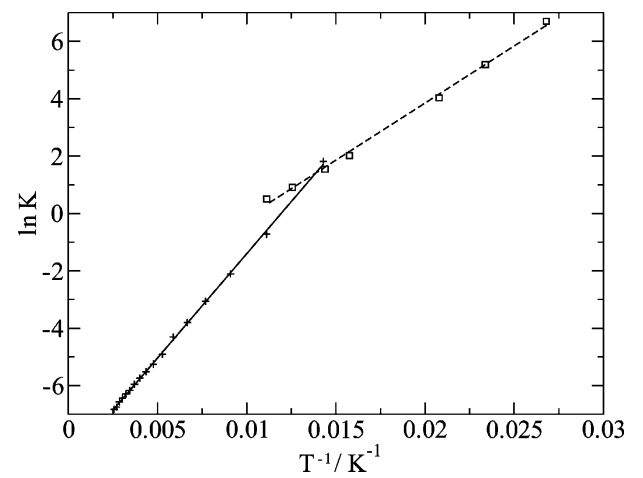

Figure 3. The natural logarithm of the Langmuir constant from pressure measurements and EMD simulation ${ }^{41,42}$ plotted versus the inverse of temperature. The simulation data are plotted as plus signs and the experimental data are plotted as squares.

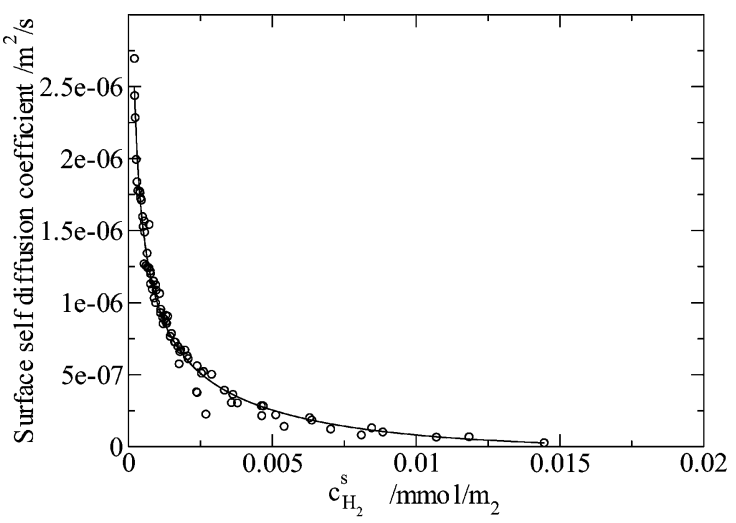

(a) EMD values of $D_{\|}^{s}$

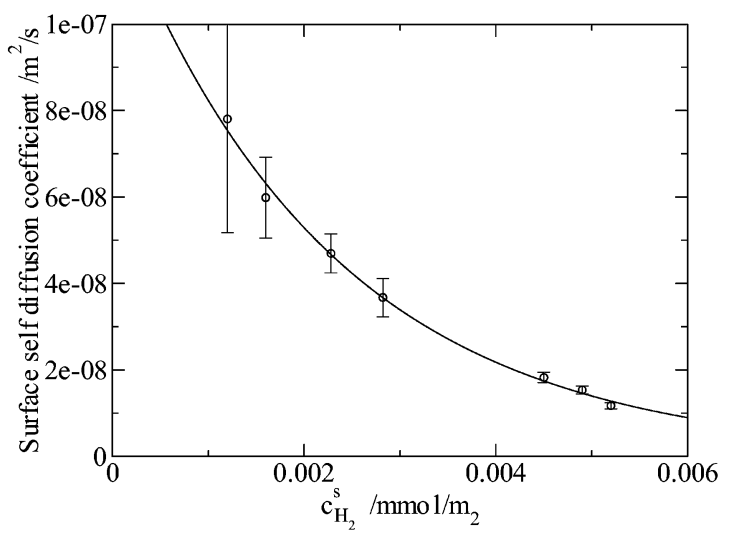

(b) QENS values of $D_{\|}^{s}$

Figure 4. (a) $D_{\|}^{\mathrm{s}}$ for all temperatures simulated with EMD plotted as function of $c_{\mathrm{H}_{2}}^{\mathrm{s}}$. The $D_{\|}^{\mathrm{s}}$ values are following the same trend for all temperatures. The solid line in both plots are guides to the eye. (b) The change in $D_{\|}^{\mathrm{s}}$ as a function of $c_{\mathrm{H}_{2}}^{\mathrm{s}}$ as found by QENS.

surface concentration are large and explain the discrepancy of the data. At high surface densities, the diffusion coefficient increases only slightly with temperature at a given density. From kinetic theory it is also predicted that $D_{\|}^{\mathrm{s}}$ increases linearly with the square root of the temperature for a given concentration. This was not observed in our data. In Figure $5 \mathrm{~b}$ we plotted the logarithm of $D_{\|}^{\mathrm{s}}$ as a function of the inverse of temperature. The trend is linear as is observed experimentally for a constant number of molecules in the system. QENS results are also plotted in the same figure and can be directly compared with simulation data at 90 and $70 \mathrm{~K}$. The EMD diffusion coefficients ranged between $7 \times 10^{-8}$ and $4 \times 10^{-7} \mathrm{~m}^{2} / \mathrm{s}$ for densities respectively between 0.012 and $0.0023 \mathrm{mmol} / \mathrm{m}^{2}$ at $90 \mathrm{~K}$, and 


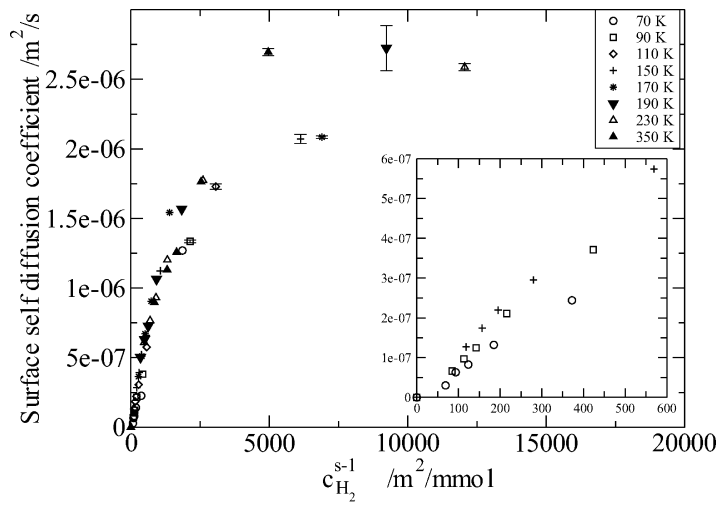

(a) $D_{\|}^{s}$ as a function of $c_{H_{2}}^{s-1}$

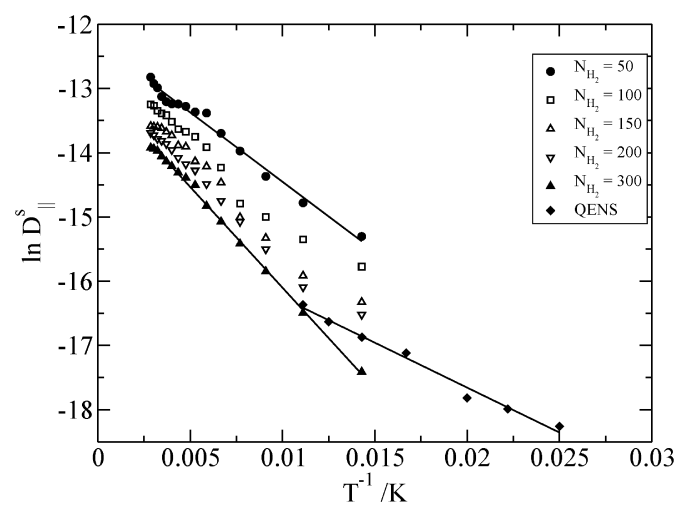

(b) Natural logarithm of $D_{\|}^{s}$

Figure 5. (a) $D_{\|}^{\text {s }}$ plotted versus the inverse of the surface concentration, $c_{\mathrm{H}_{2}}^{\mathrm{S}}{ }^{-1}$, a zoom of the high density part is also shown and reveals a temperature effect. (b) Logarithm of $D_{\|}^{\mathrm{s}}$ versus $T^{-1}$ for the EMD data, and QENS data for the 0.5 monolayer loading.

at $70 \mathrm{~K}$ they ranged between $3 \times 10^{-8}$ and $2 \times 10^{-7} \mathrm{~m}^{2} / \mathrm{s}$ for densities respectively between 0.014 and $0.0027 \mathrm{mmol} / \mathrm{m}^{2}$. The extrapolation of EMD diffusion coefficients to experimental surface densities at 70 and $90 \mathrm{~K}$, Table 1, led to higher results by about a factor of 5 . The activation energies calculated from these curves are higher by about a factor of 2 compared to the experimental results. The extrapolation of experimental data to $350 \mathrm{~K}$ gives values for the $D_{\|}^{\mathrm{S}}$ that are also 4 to 10 times lower than the EMD values. The measure of diffusion coefficients in heterogeneous systems is still a challenging topic; depending on the experimental methods the results can be several orders of magnitude different, see for example Jobic et al. ${ }^{50}$ Supporting our findings, Jobic et al. also found larger diffusivities in EMD simulations compared to QENS experiments and other experiments for alkanes in zeolites. Our EMD results are of the same magnitude as QENS data and have the same trends although EMD simulations give an ideal picture of reality. Grooves, steps, and pores in a material cause barriers for free translation for adsorbed molecules ${ }^{51,52}$ and are absent in the simulated system. This can explain the discrepancy in the activation energy and then in the data.

Reactant Transport via Supporting Surface. We have seen above that the activation energies for surface diffusion of hydrogen are about three times lower than the enthalpy of adsorption, both for EMD and QENS. This is in agreement with previous results from the literature both from theory and experiments. ${ }^{16,28,40,43-48,53,54}$ In these reports the adsorption energies are 3 to 9 times larger than the diffusion activation energy. This indicates that hydrogen molecules can be mobile at the graphite surface without escaping into the gas phase. At

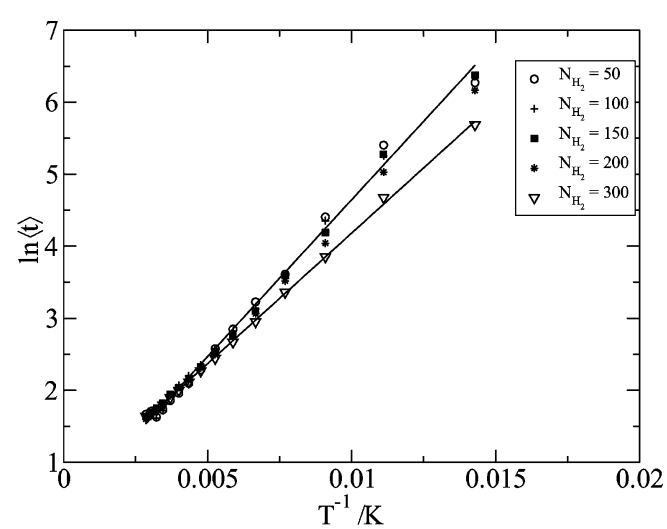

(a) Natural logarithm of $\langle t\rangle$

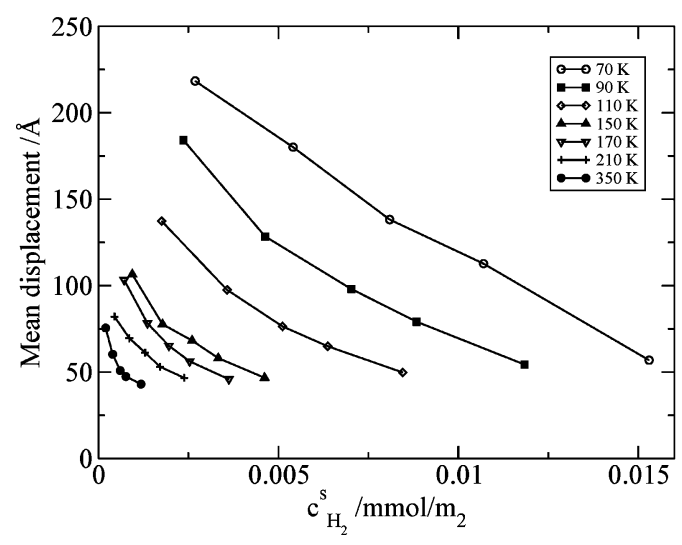

(b) $r_{\text {mean }}$ as a function of $c_{H_{2}}^{s}$

Figure 6. (a) Logarithm of the average time between adsorption and desorption events versus $T^{-1}$ for all investigated temperatures. (b) $r_{\text {mean }}$ as a function of $c_{\mathrm{H}_{2}}^{\mathrm{s}}$ for seven temperatures.

room temperature this effect is mostly assumed to be negligible. The simulation data offer a means to further investigate this assumption, from low temperatures to room temperature.

The average time, $\langle t\rangle$, between adsorption and desorption events was determined in the simulations. In Figure 6a the $\langle t\rangle$ values for each global density $N_{\mathrm{H}_{2}}$ are given as the natural logarhitm versus $T^{-1}$. The average time follows an exponential trend as given by Clark, ${ }^{55}$ and the $\langle t\rangle$ values decrease with temperature, which is in agreement with the isotherms we observed in our earlier EMD study. ${ }^{41,42}$

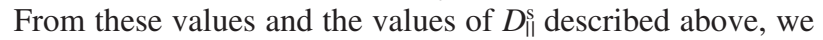
determined the mean displacement as $r_{\text {mean }}=(4 D \$\langle t\rangle)^{1 / 2}$. In Figure $6 \mathrm{~b} r_{\text {mean }}$ is plotted as a function of the surface concentration. The plot shows that $r_{\text {mean }}$ is decreasing with both increasing temperature and surface concentration. From an experimental point of view it is preferable to give these data as a function of gas pressure, see Figure 7a. In this figure $r_{\text {mean }}$ is decreasing with increasing pressure for a given temperature. At low pressure $r_{\text {mean }}$ is decreasing with increasing temperature, while at high pressure the inverse happens. This means that there exists an intermediate zone where the trends cross. This result is not in contradiction with the trend in Figure 6b. This is a consequence of the shape of the isotherms in this figure.

Extrapolation and interpolation of $r_{\text {mean }}$ as a function of pressure was used to estimate the mean displacement at $1 \mathrm{bar}$, which is a usual operating pressure of PEMFCs. The results are given in Figure $7 \mathrm{~b}$ for temperatures between 70 and $350 \mathrm{~K}$ at 1 bar. This curve shows a maximum at $110 \mathrm{~K}$ of about 140 $\AA$. This maximum can be understood from the temperature 


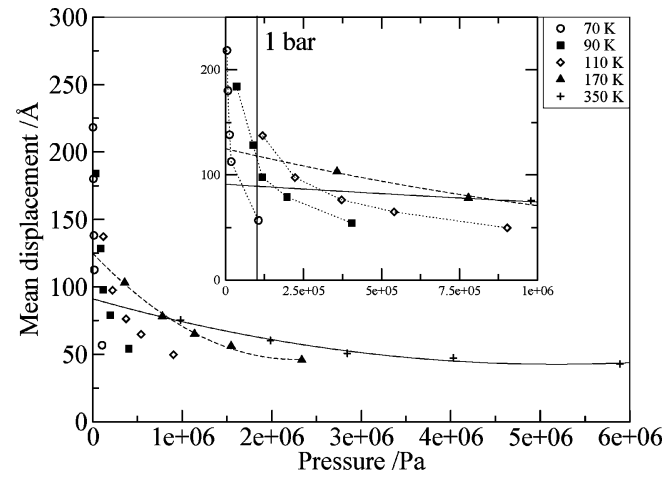

(a) $r_{\text {mean }}$ as function of pressure

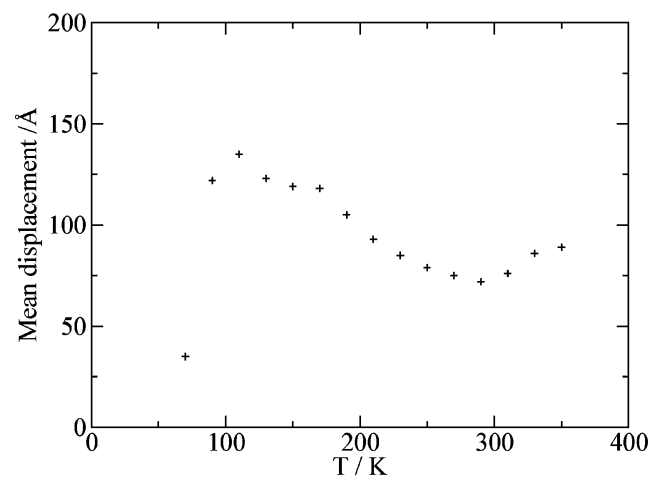

(b) Mean displacement at 1 bar

Figure 7. (a) $r_{\text {mean }}$ as function of pressure at constant temperature; the dashed line shows an extrapolation of the data at $170 \mathrm{~K}$, the full line shows an extrapolation of the data at $350 \mathrm{~K}$, the vertical line in the zoom shows the position of 1 bar $(101325 \mathrm{~Pa})$. (b) Mean displacement of the adsorbed hydrogen molecules at 1 bar for 70 to $350 \mathrm{~K}$.

dependence in $D_{\|}^{\mathrm{s}}$ and the average time. Below this temperature, the effect of $D_{\|}^{\mathrm{S}}$ is dominating and the $r_{\text {mean }}$ is increasing with temperature. Above $110 \mathrm{~K}$ it is the average time that is dominating, and $r_{\text {mean }}$ is decreasing with increasing temperature. The increase above $290 \mathrm{~K}$ is not significant. It is worthwhile to notice that even at $350 \mathrm{~K}$ the $r_{\text {mean }}$ is comparable to the lower temperature values of about $80 \AA$. Thus, the assumption that surface diffusion is negligible is doubtful, and we will in the following investigate this effect in PEMFC electrodes.

The effect of the catalyst support surface can now be investigated, by calculating the flux of reactants to a catalyst particle. In a PEMFC this translates to the flux directed along the carbon surface to a platinum particle. To do this we assume that the average catalyst particle in a PEMFC has a radius $R$ of $1 \mathrm{~nm}^{56}$ and is shaped as a hemisphere. Further we assume that the flux of adsorbing gas molecules perpendicular to the carbon surface is uniform.

The area of the catalyst support material around the catalyst, with an influx of reactants, is equal to the area of a circle with radius equal to $r_{\text {mean }}+R$, minus the area of the catalyst particle. This is given by

$$
A_{\text {support }}=\pi\left(r_{\text {mean }}+R\right)^{2}-\pi R^{2}=\pi\left(r_{\text {mean }}^{2}+2 r_{\text {mean }} R\right)
$$

This area is hereinafter referred to as the active support area. For a given temperature, the active support area is constant and it is equal to the area of the large circle minus the area of the

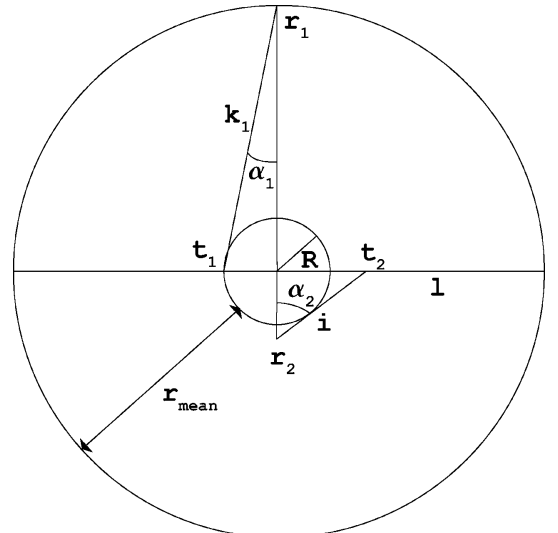

Figure 8. Geometry defining the active support surface and the angle, $\alpha_{i}$, giving the probability of adsorbed molecules reaching the catalyst particle.

inner circle in Figure 8. To estimate how many of the molecules that adsorb on the active support area travel to the catalyst particle, we have to define the probability of an adsorbed molecule hitting the catalyst particle. This probability is a function of the distance from the catalyst particle where the molecules adsorb. In Figure 8 two examples of two different adsorptions, at positions $r_{1}$ and $r_{2}$, are shown. Assuming that the adsorbed molecules are just as likely to move in all directions after adsorbing at $r_{i}$, and desorb first when they are outside a circle with radius $r_{\text {mean }}$, the probability $p_{i}$ can be given by the angle $\alpha_{i}$, distance from the catalyst particle $r_{i}$, and the position $t_{i}$ on line $l$. The probability of adsorbed molecules reaching the catalyst particle in an arbitrary position is then

$$
p\left(r_{i}, t_{i}\right)=\frac{2 \alpha_{i}}{2 \pi}=\frac{2 \arctan \left(t_{i} / r_{i}\right)}{2 \pi}=\frac{\arctan \left(t_{i} / r_{i}\right)}{\pi}
$$

where $r_{i}$ is an arbitrary position within the active support area of the catalyst support and $t_{i}$ is the position on line $l$ where line $k$ intersects, see Figure 8. Since the position $t_{i}$ changes with the position $r_{i}$ we can give a function describing $t_{i}$ with $R$ and $r_{i}$. This function is given by the functions of a circle and a line segment $k$. We know that these two functions are equal where they meet at the intersection $i$, and that the derivatives also are equal there. We then find the function for the position $t_{i}$ as

$$
t_{i}\left(r_{i}, R\right)=\frac{R}{\sqrt{\left(1-\frac{R^{2}}{r_{i}^{2}}\right)}}
$$

When we introduce this in eq 15 we get

$$
p_{i}\left(r_{i}\right)=\arctan \left(\left[\frac{R}{\sqrt{\left(1-\frac{R^{2}}{r_{i}^{2}}\right)}}\right] / r_{i}\right) / \pi
$$

To find the fraction of molecules adsorbed on the active support area that reach the catalyst particle, we integrate for the whole surface and normalize by the active support area: 


$$
\begin{gathered}
P=\frac{1}{A_{\text {support }}} \int_{R}^{r_{i}} \int_{0}^{2 \pi} p\left(r_{i}, \phi\right) r_{\text {mean }} \mathrm{d} \phi \mathrm{d} r \\
P=\frac{1}{\pi\left(r_{\text {mean }}^{2}+2 r_{\text {mean }} R\right)} \times \\
{\left[\left(\arctan \left(\frac{R}{\sqrt{1-\frac{R^{2}}{r_{i}^{2}}} r_{\text {mean }}}\right) r_{\text {mean }}^{2}+R \sqrt{1-\frac{R^{2}}{r_{i}^{2}}} r_{\text {mean }}\right)\right]_{R}^{r_{\text {mean }}}}
\end{gathered}
$$

Here $P$ is the fraction of molecules adsorbed on the active support surface and reaching the catalyst particle. Since the adsorption flux is uniform this allows us to calculate the ratio between the flux from the gas phase directly to the platinum, $J_{\text {gas }}$, and the flux from the active support area, $J_{\text {support }}$, to the catalyst particle. The ratio between the fluxes is proportional to the active support area and the catalyst particle area and is given as

$$
\begin{array}{r}
\frac{J_{\text {support }}}{J_{\text {gas }}}=\frac{A_{\text {support }} P}{A_{\text {catalyst }}}=\frac{\pi\left(r_{\text {mean }}^{2}+2 r_{\text {mean }} R\right) P}{2 \pi R^{2}}= \\
\frac{\left(r_{\text {mean }}^{2}+2 r_{\text {mean }} R\right) P}{2 R^{2}}
\end{array}
$$

The results of eqs 18 and 19 are given in Figures 9, panels a and b, with $R$ equal $1 \mathrm{~nm}$ and a mean displacement up to 8 $\mathrm{nm}$. In Figure $9 \mathrm{~b}$ the ratio between $J_{\text {support }}$ and $J_{\text {gas }}$ is plotted. From Figure $9 \mathrm{~b}$ and the values from Figure $7 \mathrm{~b}$ we can find the contribution from the graphite support at 1 bar at different temperatures.

The results from the EMD simulation were used to estimate the amount of reactant that can be transported to a catalyst particle in a PEMFC. Figure $9 \mathrm{~b}$ gives the ratio of the reactant transported via the active support area, $J_{\text {support }}$, and the reactant adsorbed from the gas phase, $J_{\text {gas }}$, plotted as a function of the mean displacement. We saw in the previous section that $D_{\|}^{\mathrm{s}}$ from

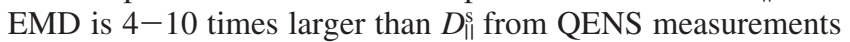
at $350 \mathrm{~K}$. We use $D_{\|}^{\mathrm{s}}=1.4 \times 10^{-7} \mathrm{~m}^{2} / \mathrm{s}$ as estimated from the QENS experiment at the highest hydrogen loading as the most realistic value, and calculate a $J_{\text {support }} / J_{\text {gas }}$ ratio close to 1 . The transport of reactants to the catalyst via the active surface area according to this is $50 \%$ of the total amount.

In the actual PEMFC, the platinum metal catalyst is in contact with the graphite support and the polymer membrane. Only a small fraction of the catalyst may have an interface in common with the gas phase. Hydrogen molecules in the gas phase might well have direct access to only a few of the active sites on the platinum catalyst. To reach the active sites, hydrogen must adsorb on platinum, graphite, or polymer membrane and then move to the site by diffusion. Hydrogen molecules on graphite surfaces must pass some three-phase contact line before they arrive at the active sites. Gas molecules cannot go directly to a three-phase contact line from the gas phase as this would lead to an infinite velocity of the gas close to the three-phase contact line. ${ }^{10}$ If the surface area of platinum in contact with the gas phase is only a fraction of the active surface area of the graphite, the ratio $J_{\text {support }} / J_{\text {gas }}$ can be much larger than estimated here. The catalytic reaction also depends on protons reaching the polymer membrane. Only then will hydrogen be transported away from the catalyst, allowing for further reaction. The diffusivity of hydrogen on platinum is in the order of $10^{-9} \mathrm{~m}^{2} / \mathrm{s},{ }^{57} 3$ orders

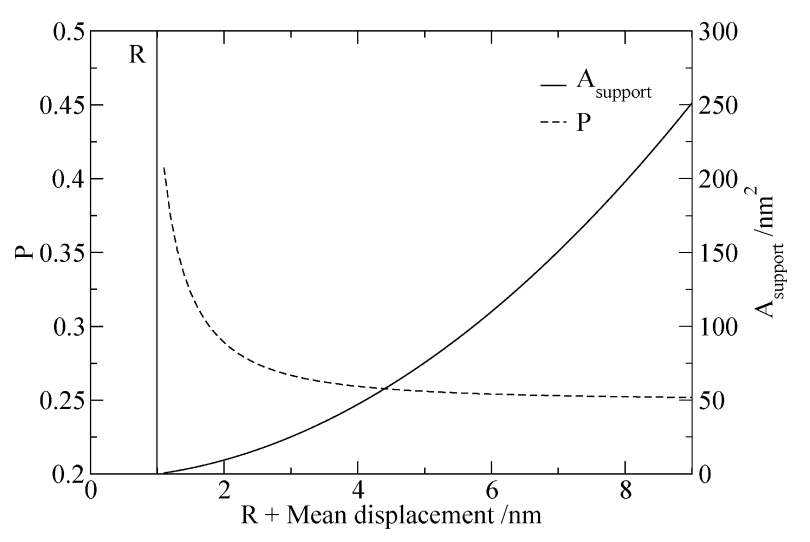

(a) Values of $P$ and $A_{\text {support }}$

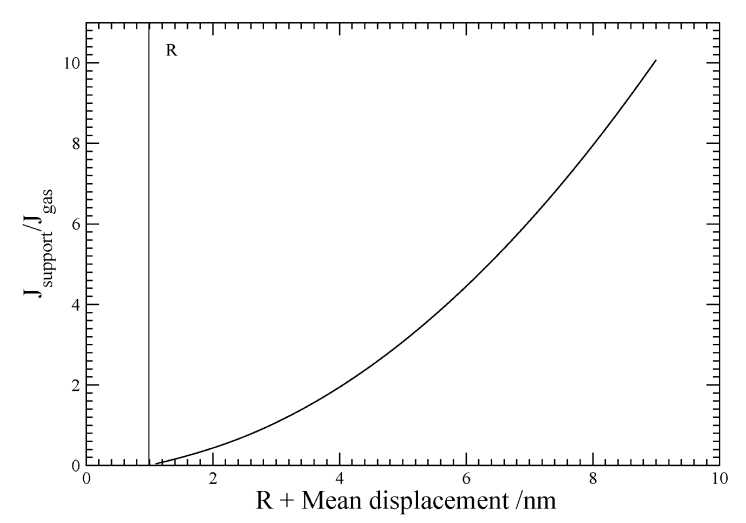

(b) Ratio of $\mathrm{J}_{\text {support }} / \mathrm{J}_{\text {gas }}$

Figure 9. (a) The fraction $P$ from eq 18 and $A_{\text {support }}$ as functions of the mean displacement plus $R$. (b) Ratio between the reactant flux from the active surface area and from the gas phase to the catalytic particle.

of magnitude smaller than that on graphite. The interface between catalyst and the polymer membrane should therefore be as close to the graphite surface as possible in order to increase the reaction rate.

\section{Conclusion}

From QENS and EMD surface self-diffusion coefficients for hydrogen adsorbed on graphite have been found. The two methods give values in good agreement for low temperatures $(90 \mathrm{~K})$, but at high temperatures EMD gives $D_{\|}^{\mathrm{s}}$ values that are 4 to 10 times larger. Both the QENS data and the EMD data

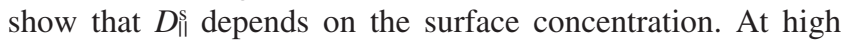
surface concentrations $D_{\|}^{\mathrm{s}}$ follows a linear trend according to kinetic theory. At low surface densities $D_{\|}^{\mathrm{s}}$ reaches a maximum value at each temperature. From the simulations the average times between adsorption and desorption events were calculated. They followed an exponential trend, increasing with temperature. From the average time and surface diffusion from EMD and QENS the mean displacement was calculated. At 1 bar, the mean displacement had a maximum value at $110 \mathrm{~K}$ of about $140 \AA$. For ambient temperature and pressure the value was about 80 $\AA$. These results have been used to estimate the transport of reactants via the catalyst support material in the PEMFC to a catalyst particle. From these estimates the catalyst support is responsible for at a minimum $50 \%$ of the reactant transport. This suggests that the catalyst support is contributing considerably to the reactant transport in the PEMFC.

Acknowledgment. For experimental assistance and much appreciated help we wish to thank Peter Fouquet, Hannu Mutka, 
and Stéphane Rols at the Institut Laue-Langevin, Grenoble. The authors also thank Leen van der Ham for nice discussions. Financial support from the Norwegian Research Council, Storforsk (grant no. 167336/V30) is much appreciated.

\section{References and Notes}

(1) Pozio, A.; De Francesco, M.; Cemmi, A.; Cardellini, F.; Giorgi, L. J. Power Sources 2002, 105, 13-19.

(2) Ismagilov, Z. R.; Kerzhentsev, M. A.; Shikina, N. V.; Lisitsyn, A. S.; Okhlopkova, L. B.; Barnakov, C.; Sakashita, M.; Iijima, T.; Tadokoro, K. Catal. Today 2005, 102-103, 58-66.

(3) Larminie, J.; Dicks, A. Fuel Cell Systems Explained, 2nd ed.; John Wiley \& Sons: New York, 2003. 252.

(4) Costamagna, P.; Srinivasan, S. J. Power Sources 2001, 102, 242-

(5) Stevens, D. A.; Zhang, S.; Chen, Z.; Dahn, J. R. Carbon 2003, 41, $2769-2777$.

(6) Passos, R. R.; Paganin, V. A.; Ticianelli, E. A. Electrochim. Acta 2006, 51, 5239-5245.

(7) Baschuk, J. J.; Li, X. J. Power Sources 2005, 142, 134-153.

(8) Gode, P.; Jaouen, F.; Lindbergh, G.; Lundblad, A.; Sundholm, G. Electrochim. Acta 2003, 48, 4175-4187.

(9) Rajalakshmi, N.; Dhathathreyan, K. Int. J. Hydrogen Energy 2008, $33,5672-5677$.

(10) Kjelstrup, S.; Bedeaux, D. Non-Equilibrium Thermodynamics of Heterogeneous Systems; World Scientific Publishing Co. Pte, Ltd.: Singapore, 2008.

(11) Meland, A. K.; Bedeaux, D.; Kjelstrup, S. J. Phys. Chem. B 2005, 109, 21380-21388.

(12) Meland, A. K.; Kjelstrup, S.; Bedeaux, D. J. Membr. Sci. 2006, 282, 96-108.

(13) Paulus, U. A.; Veziridis, Z.; Schnyder, B.; Kuhnke, M.; Scherer, G. G.; Wokaun, A. J. Electroanal. Chem. 2003, 541, 77-91.

(14) Haas, O. E.; Simon, J. M.; Kjelstrup, S.; Ramstad, A. L.; Fouquet, P. J. Phys. Chem. C 2008, 112, 3121-3125.

(15) Bienfait, M.; Zeppenfeld, P.; Bovie, L. J.; Vilches, O. E.; Lauter, H. J. Phys. B (Amsterdam, Neth.) 1997, 234-236, 159-163.

(16) Narehood, D. G.; Pearce, J. V.; Eklund, P. C.; Sokol, P. E.; Lechner, R. E.; Pieper, J.; Copley, J. R. D.; Cook, J. C. Phys. Rev. B: Condens. Matter Mater. Phys. 2003, 67, 205409-5.

(17) Dillon, A. C.; Heben, M. J. Appl. Phys. A: Mater. Sci. Process. 2001, 72, 133-142.

(18) Chambers, A.; Park, C.; Baker, R. T. K.; Rodriguez, N. M. J. Phys. Chem. B 1998, 102, 4253-4256.

(19) Strobel, R.; Jorissen, L.; Schliermann, T.; Trapp, V.; Schutz, W.; Bohmhammel, K.; Wolf, G.; Garche, J. J. Power Sources 1999, 84, 221224.

(20) Benard, P.; Chahine, R. Langmuir 2001, 17, 1950-1955.

(21) Takagi, H.; Hatori, H.; Yamada, Y.; Matsuo, S.; Shiraishi, M. J. Alloys Compd. 2004, 385, 257-263.

(22) Shindo, K.; Kondo, T.; Sakurai, Y. J. Alloys Compd. 2004, 379, 252-255.

(23) Zhang, J.; Wang, X.; Wu, C.; Wang, H.; Yi, B.; Zhang, H. React. Kinet. Catal. Lett. 2004, 83, 229-236.

(24) Blackman, J. M.; Patrick, J. W.; Snape, C. E. Carbon 2006, 44, 918-927.

(25) Zhou, L.; Zhou, Y.; Sun, Y. Int. J. Hydrogen Energy 2006, 31, 259-264.

(26) Ren, Y.; Price, D. L. Appl. Phys. Lett. 2001, 79, 3684-3686.
(27) Narehood, D. G.; Kostov, M. K.; Eklund, P.; Cole, M. W.; Sokol, P. E. Phys. Rev. B 2002, 65.

(28) Schimmel, H. G.; Nijkamp, G.; Kearley, G. J.; Rivera, A.; de Jong,

K. P.; Mulder, F. M. Mater. Sci. Eng. B 2004, 108, 124-129.

(29) Georgiev, P. A.; Ross, D. K.; De Monte, A.; Montaretto-Marullo, U.; Edwards, R. A. H.; Ramirez-Cuesta, A. J.; Adams, M. A.; Colognesi, D. Carbon 2005, 43, 895-906.

(30) Hempelmann, R. Quasielastic Neutron Scattering and Solid State Diffusion; Oxford University Press: Oxford, UK, 2000.

(31) Egelstaff, P. A. An Introduction to the Liquid State; Oxford University Press: Oxford, UK, 1992.

(32) Wyckoff, R. W. G. Crystal Structures; Interscience: New York, 1963.

(33) Adri, C. T. v. D.; Jan, M. A. B.; Bastiaan van de, G. J. Chem. Soc., Faraday Trans. 1994, 90, 2881-2895.

(34) Haile, J. Molecular dynamics simulation: elementary methods; John Wiley \& Sons: New York, 1992.

(35) Nicholas, J. B.; Trouw, F. R.; Mertz, J. E.; Iton, L. E.; Hopfinger,

A. J. J. Phys. Chem. 1993, 97, 4149-4163.

(36) Mayo, S. L.; Olafson, B. D.; Goddard, W. A. J. Phys. Chem. 1990, 94, 8897-8909.

(37) Allen, M. P.; Tildesly, D. J. Computer Simulation of Liquids; Claredon Press: Oxford, UK, 1987.

(38) Rudzinski, W.; Steele, W.; Zgrablich, G. Equilibria and Dynamics of Gas Adsorption on Heterogeneous Solid Surfaces; Elsevier Science B.V.: Amsterdam, The Netherlands, 1997.

(39) O'Reilly, D. E.; Peterson, E. M. J. Chem. Phys. 1977, 66, 934937.

(40) Cabria, I.; Lopez, M. J.; Alonso, J. A. Comput. Mater. Sci. 2006, $35,238-242$.

(41) Haas, O. E.; Simon, J. M.; Kjelstrup, S. In preparation.

(42) Haas, O. E. Ph.D. thesis, Norwegian University of Science and Technology, NTNU, Norway, 2009..

(43) Nijkamp, M. G.; Raaymakers, J. E. M. J.; van Dillen, A. J.; de Jong, K. P. Appl. Phys. A: Mater. Sci. Process. 2001, 72, 619-623.

(44) Strobel, R.; Garche, J.; Moseley, P. T.; Jorissen, L.; Wolf, G. J. Power Sources 2006, 159, 781-801.

(45) Arellano, J. S.; Molina, L. M.; Rubio, A.; Alonso, J. A. J. Chem. Phys. 2000, 112, 8114-8119.

(46) Arellano, J. S.; Molina, L. M.; Rubio, A.; Lopez, M. J.; Alonso, J. A. J. Chem. Phys. 2002, 117, 2281-2288.

(47) Jacobson, N.; Tegner, B.; Schroder, E.; Hyldgaard, P.; Lundqvist, B. I. Comput. Mater. Sci. 2002, 24, 273-277.

(48) Patchkovskii, S.; Tse, J. S.; Yurchenko, S. N.; Zhechkov, L.; Heine,

T.; Seifert, G. Proc. Natl. Acad. Sci. U.S.A. 2005, 102, 10439-10444.

(49) Cracknell, R.; Nicholson, D.; Gubbins, K. J. Chem. Soc., Faraday

Trans. 1995, 91, 1377-1383.

(50) Jobic, H. J. Mol. Catal A: Chem. 2000, 158, 135-142.

(51) Steele, W. Equilibria and dynamics of gas adsorption on heterogeneous solid surfaces. In. Stud. Surf. Sci. Catal. 1997, 104, 451-485.

(52) Bojan, M. J.; Steele, W. A. Langmuir 1993, 9, 2569-2575.

(53) Young, D.; Crowell, A. Physical adsorption of gases; Buttersworth: London, UK, 1962.

(54) Bienfait, M.; Zeppenfeld, P.; Ramos, R. C.; Gay, J. M.; Vilches,

O. E.; Coddens, G. Phys. Rev. B 1999, 60, 11773.

(55) Clark, A. The Theory of adsorption and catalysis; Academic Press: New York, 1970.

(56) Stevens, D. A.; Hicks, M. T.; Haugen, G. M.; Dahn, J. R. J. Electrochem. Soc. 2005, 152, A2309-A2315.

(57) Liu, W.-J.; Wu, B.-L.; Cha, C.-S. Russ. J. Electrochem. 2000, 36, 846-851.

JP902491S 